TRANSACTIONS OF THE

AMERICAN MATHEMATICAL SOCIETY

Volume 351, Number 9, Pages 3857-3868

S 0002-9947(99)02137-6

Article electronically published on April 20, 1999

\title{
ON THE NUMBER OF TERMS IN THE MIDDLE OF ALMOST SPLIT SEQUENCES OVER TAME ALGEBRAS
}

\author{
J. A. DE LA PEÑA AND M. TAKANE
}

\begin{abstract}
Let $A$ be a finite dimensional tame algebra over an algebraically closed field $k$. It has been conjectured that any almost split sequence $0 \rightarrow$ $X \rightarrow \oplus_{i=1}^{n} Y_{i} \rightarrow Z \rightarrow 0$ with $Y_{i}$ indecomposable modules has $n \leq 5$ and in case $n=5$, then exactly one of the $Y_{i}$ is a projective-injective module. In this work we show this conjecture in case all the $Y_{i}$ are directing modules, that is, there are no cycles of non-zero, non-iso maps $Y_{i}=M_{1} \rightarrow M_{2} \rightarrow \cdots \rightarrow M_{s}=Y_{i}$ between indecomposable $A$-modules. In case, $Y_{1}$ and $Y_{2}$ are isomorphic, we show that $n \leq 3$ and give precise information on the structure of $A$.
\end{abstract}

Let $A$ be a finite dimensional algebra over an algebraically closed field $k$. We denote by $\bmod _{A}$ the category of finite dimensional left $A$-modules (an object in $\bmod _{A}$ is simply called a module).

For a non-projective indecomposable module $X$, there exist an indecomposable non-injective module $\tau_{A} X$ called the Auslander-Reiten translate and an almost split sequence $0 \rightarrow \tau_{A} X \rightarrow E \rightarrow X \rightarrow 0$ (see [2], [18]). Since their introduction, almost split sequences have played a central role in the representation theory of algebras (see for example [2]).

For an almost split sequence $0 \rightarrow \tau_{A} X \rightarrow E \rightarrow X \rightarrow 0$, consider the indecomposable decomposition $E=\bigoplus_{i=1}^{s(X)} Y_{i}$. There has been considerable attention paid to the relation between properties of the algebra $A$ and the values $s(X)$ for different modules $X$ (and of course between properties of $X$ and the value $s(X)$ ). Among other interesting results we recall that if $A$ is representation finite, then $s(X) \leq 4$, for every indecomposable non-projective module $X$ [3], [7] (see also [10] and [12]).

It has been conjectured by S. Brenner that, for $A$ a tame algebra, $s(X) \leq 5$ for every indecomposable non-projective module $X$. This is known to hold for many examples, in particular for the important case of hereditary tame algebras.

To state the main results of this work, we recall some concepts. A cycle in $\bmod _{A}$ is a sequence $X_{0} \stackrel{f_{1}}{\longrightarrow} X_{1} \stackrel{f_{2}}{\longrightarrow} \quad \cdots \quad \stackrel{f_{s}}{\longrightarrow} X_{s}=X_{0}$ of non-zero nonisomorphism maps between indecomposable modules; the cycle is said to be finite if $f_{i} \notin \operatorname{rad}_{A}^{\infty}\left(X_{i-1}, X_{i}\right)$, for all $i=1, \ldots, s$. An indecomposable module $X$ is said to be directing if it does not belong to a cycle in $\bmod _{A}$. The algebra $A$ is said to be cycle-finite if all cycles in $\bmod _{A}$ are finite. We recall that a cycle finite algebra is tame [1].

The Coxeter matrix $\phi_{A}$ of $A$ and its spectral radius $\rho\left(\phi_{A}\right)=\max \{\|\lambda\|: \lambda$ eigenvalue of $\left.\phi_{A}\right\}$ are important invariants (see for example [5], [14], [17], [20]). In case

Received by the editors August 22, 1996 and, in revised form, April 25, 1997.

1991 Mathematics Subject Classification. Primary 16G60, 16G70. 
$A$ is a triangular algebra, we denote by

$$
\tilde{\rho}(A)=\max \left\{\rho\left(\phi_{B}\right): B=A / A e A \text { for some idempotent } e \in A\right\} .
$$

Theorem 1. Assume that $A$ is a triangular algebra such that

$$
\tilde{\rho}(A) \leq \frac{t-2+\sqrt{t^{2}-4 t}}{2}
$$

for some natural number $t \geq 4$. Let $0 \rightarrow \tau_{A} X \rightarrow \bigoplus_{i=1}^{s(X)} Y_{i} \rightarrow X \rightarrow 0$ be an almost split sequence in $\bmod _{A}$ such that $Y_{i}$ is directing $(i=1, \ldots, s(X))$. Then $s(X) \leq t+1$.

Theorem 2. Assume $A$ is a tame algebra and let $0 \rightarrow \tau_{A} X \rightarrow \bigoplus_{i=1}^{s(X)} Y_{i} \rightarrow X \rightarrow 0$ be an almost split sequence in $\bmod _{A}$ such that $Y_{i}$ is directing $(i=1, \ldots, s(X))$.

Then the following holds:

(a) $s(X) \leq 5$. Moreover, if $s(X)=5$, then for some $j \in\{1, \ldots, s(X)\}$, the module $Y_{j}$ is projective and injective.

(b) If $s(X) \geq 3$, and $Y_{1} \cong Y_{2}$, then $s(X)=3$ and the module $Y_{3}$ is projective and injective.

Theorem 3. Assume $A$ is a cycle-finite algebra. Then for any almost split sequence $0 \rightarrow \tau_{A} X \rightarrow \bigoplus_{i=1}^{s(X)} Y_{i} \rightarrow X \rightarrow 0$ we have $s(X) \leq 5$. If $s(X)=5$, there is some $Y_{j}(1 \leq j \leq s)$ which is projective and injective.

We prove the theorems in section 2 after some preliminary considerations. We gratefully acknowledge support of CONACYT and DGAPA, UNAM.

\section{Cycles and almost split sequences}

1.1. Let $H=k \Delta$ be the path algebra of a quiver $\Delta$ without oriented cycles (see [6]). A tilting module $T$ in $\bmod _{H}$ satisfies: $\operatorname{Ext}_{H}^{1}(T, T)=0$ and there is an exact sequence $0 \rightarrow H \rightarrow T^{\prime} \rightarrow T^{\prime \prime} \rightarrow 0$ with $T^{\prime}, T^{\prime \prime} \in \operatorname{add} T$ (see [18]). For a tilting module ${ }_{H} T$, the algebra $B=\operatorname{End}_{H}(T)$ is called a tilted algebra of type $\Delta$.

We recall that an indecomposable $B$-module $X$ is sincere if $\operatorname{Hom}_{B}(P, X) \neq 0$ for every projective $B$-module $P$. If $X$ is a sincere directing indecomposable $B$-module, then $B$ is a tilted algebra $[18$, p. 375$]$.

1.2. The Auslander-Reiten quiver $\Gamma_{A}$ of $A$ has as vertices representatives of the iso-classes of indecomposable modules; there are as many arrows $X \rightarrow Y$ in $\Gamma_{A}$ as $\operatorname{dim}_{k} \operatorname{rad}_{A}(X, Y) / \operatorname{rad}_{A}^{2}(X, Y)$. A path $Y_{0} \rightarrow Y_{1} \rightarrow \cdots \rightarrow Y_{s}$ in $\Gamma_{A}$ is said to be sectional if $\tau_{A} Y_{i+1} \neq Y_{i-1}$ for all $i=1, \ldots, s-1$. In case $Y_{0}=Y_{s}$, we have a sectional cycle if $\tau_{A} Y_{i+1} \neq Y_{i-1}$ for all $i=1, \ldots, s$ mod $s$. A sectional path in $\Gamma_{A}$ contains no sectional cycle [4].

A component $\mathcal{C}$ of $\Gamma_{A}$ is directing if it is formed by directing modules.

1.3. Let $\eta: 0 \rightarrow \tau_{A} X \rightarrow \bigoplus_{i=1}^{s(X)} Y_{i} \rightarrow X \rightarrow 0$ be an almost split sequence in $\bmod _{A}$ and let $B(\eta)$ be a quotient $A / A e A$, with $e$ idempotent in $A$, of minimal dimension such that $\eta$ is formed by $B(\eta)$-modules. By [18], one of the modules $\tau_{A} X, X$ or $Y_{i}(1 \leq i \leq s(X))$ is sincere as $B(\eta)$-module. 
Lemma. Let $\eta: 0 \rightarrow \tau_{A} X \rightarrow \bigoplus_{i=1}^{s(X)} Y_{i} \rightarrow X \rightarrow 0$ be an almost split sequence in $\bmod _{A}$ such that $Y_{i}$ is directing as $B(\eta)$-module, for $i=1, \ldots, s(X)$. Then

(a) $X$ and $\tau_{A} X$ are directing as $B(\eta)$-modules;

(b) $B(\eta)$ is a tilted algebra.

Proof. Consider the almost split sequence $\eta: 0 \rightarrow \tau_{A} X \stackrel{\left(h_{i}\right)}{\longrightarrow} \bigoplus_{i=1}^{s(X)} Y_{i} \stackrel{\left(g_{i}\right)}{\longrightarrow} X \rightarrow 0$. Let $X=Z_{0} \stackrel{f_{1}}{\longrightarrow} Z_{1} \rightarrow \cdots \rightarrow Z_{s-1} \stackrel{f_{s}}{\longrightarrow} Z_{s}=X$ be a cycle in $\bmod _{B(\eta)}$. The map $f_{s}: Z_{s-1} \rightarrow Z_{s}=X$ factorizes through the sink map $\left(g_{i}\right)_{i}: \bigoplus_{i=1}^{s(X)} Y_{i} \rightarrow X$ and for some $j \in\{1, \ldots, s(X)\}$ we get a non-zero map $f_{s}^{\prime}: Z_{s-1} \rightarrow Y_{j}$. Therefore we get a cycle

$$
Y_{j} \stackrel{g_{j}}{\longrightarrow} X=Z_{0} \stackrel{f_{1}}{\longrightarrow} Z_{1} \rightarrow \cdots \rightarrow Z_{s-1} \stackrel{f_{s}^{\prime}}{\longrightarrow} Y_{j} .
$$

A contradiction. Hence $X$ (and similarly $\tau_{A} X$ ) is directing. The algebra $B(\eta)$ is tilted by (1.1).

1.4 Proposition. Let $X$ be a directing indecomposable non-projective module and $\eta: 0 \rightarrow \tau_{A} X \rightarrow \bigoplus_{i=1}^{s(X)} Y_{i} \rightarrow X \rightarrow 0$ be the corresponding almost split sequence. Assume $s(X) \geq 3$. Then one of the following conditions holds:

(a) $B(\eta)$ is a tilted algebra and $X$ belongs to a directing component of $\Gamma_{B(\eta)}$;

(b) there exists a sectional path $\tau_{A} X=Z_{0} \rightarrow Z_{1} \rightarrow \cdots \rightarrow Z_{s}$ in $\Gamma_{B(\eta)}$ with $Z_{s}$ an injective module.

Proof. We shall denote $B=B(\eta)$. Assume first that $Y_{i}$ is directing as $B$-module for $i=1, \ldots, s(X)$. By (1.3), $B$ is a tilted algebra. By [9], we know the structure of $\Gamma_{B}$ : there is a postprojective, a preinjective and a connecting component (some of these components may coincide) and components of type $\mathbb{Z} \mathbb{A}_{\infty}$ or $\mathbb{Z} \mathbb{A}_{\infty} /(n)$, possibly with inserted ray modules or coinserted coray modules. Since $s(X) \geq 3$, then $X$ belongs to a postprojective, preinjective or connecting component of $\Gamma_{B}$, all of which are directing. Hence (a) holds.

Assume $Y_{1}$ is not directing. Let $Y_{1}=Z_{0} \stackrel{f_{1}}{\longrightarrow} Z_{1} \rightarrow \cdots \stackrel{f_{s}}{\longrightarrow} Z_{s}=Y_{1}$ be a cycle in $\bmod _{B}$. By (1.2), we may assume that one of the following situations occurs:

(1) $Y_{1}=Z_{0} \stackrel{f_{1}}{\longrightarrow} Z_{1} \rightarrow \cdots \stackrel{f_{r}}{\longrightarrow} Z_{r} \stackrel{f_{r+1}}{\longrightarrow} Z_{r+1} \rightarrow \cdots \rightarrow Y_{1}$ such that $Z_{0} \stackrel{f_{1}}{\longrightarrow} Z_{1} \rightarrow \cdots \stackrel{f_{r}}{\longrightarrow} Z_{r}$ is a sectional path in $\Gamma_{B}$ and $\tau_{B} Z_{r+1}=Z_{r-1}$;

(2) for all $i \in \mathbb{N}$, there is a sectional path in $\Gamma_{B}, Y_{1}=Z_{0} \stackrel{f_{1}}{\longrightarrow} Z_{1} \rightarrow \cdots \stackrel{f_{i}}{\longrightarrow} Z_{i}$ and a path $Z_{i} \rightarrow Z_{i+1}^{\prime} \rightarrow \cdots \rightarrow Y_{1}$ in $\bmod _{B}$.

If some $Z_{i}$ is injective $(1 \leq i \leq r$ in case (1) or $1 \leq i$ in case (2)), then (b) holds. We assume that no $Z_{i}$ is injective in order to get a contradiction.

First observe that situation (1) cannot happen. Otherwise, we get a cycle

$$
Y_{1} \stackrel{h_{1}}{\longrightarrow} X \rightarrow \tau_{B}^{-} Y_{1} \rightarrow \tau_{B}^{-} Z_{1} \rightarrow \cdots \tau_{B}^{-} Z_{r-1}=Z_{r+1} \rightarrow \cdots \rightarrow Y_{1} \stackrel{h_{1}}{\longrightarrow} X,
$$

where $h_{1}: Y_{1} \rightarrow X$ is an irreducible map. Contradicting that $X$ is a directing $A$-module. 
Let $n$ be the number of iso-classes of simple $A$-modules and consider a sectional path $Y_{1}=Z_{0} \stackrel{f_{1}}{\longrightarrow} Z_{1} \stackrel{f_{2}}{\longrightarrow} Z_{2} \rightarrow \cdots \stackrel{f_{n}}{\longrightarrow} Z_{n}$ in $\Gamma_{B}$ as given in situation (2). Moreover, there is a path $Z_{n} \rightarrow Z_{n+1}^{\prime} \rightarrow \cdots \rightarrow Y_{1}$ in $\bmod _{B}$. We shall prove that $\bigoplus_{i=0}^{n} Z_{i}$ is a partial cotilting module, which yields the desired contradiction. Indeed,

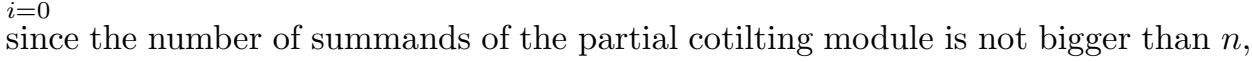
then we get $Z_{i} \cong Z_{j}$ for some $j>i$. By (1.2), the cycle $Z_{i} \rightarrow \cdots Z_{j-1} \rightarrow Z_{j}$ is not sectional and hence $Z_{j-1} \cong \tau_{B} Z_{i+1}$ or $Z_{j-2} \cong \tau_{B} Z_{i}$ which yields a cycle through $X$ as above.

Let us first show that $i \operatorname{dim}_{B} Z_{i} \leq 1, i=0, \ldots, n$. Otherwise $i \operatorname{dim}_{B} Z_{i}>1$ and there are an indecomposable projective $B$-module $P$ and a map $0 \neq g \in$ $\operatorname{Hom}_{B}\left(\tau_{B}^{-} Z_{i}, P\right)$ (see [18]). Since $\bigoplus_{i=1}^{s(X)} Y_{i}$ is a sincere $B$-module, there are some $j \in\{1, \ldots, s(X)\}$ and a map $0 \neq g^{\prime} \in \operatorname{Hom}_{B}\left(P, Y_{j}\right)$. We get a cycle

$$
X \rightarrow \tau_{B}^{-} Y_{j} \rightarrow \cdots \rightarrow \tau_{B}^{-} Z_{i} \stackrel{g}{\rightarrow} P \stackrel{g^{\prime}}{\rightarrow} Y_{j} \stackrel{h_{j}}{\longrightarrow} X,
$$

again a contradiction.

Let $i, j \in\{0, \ldots, n\}$; we show that $\operatorname{Ext}_{B}^{1}\left(Z_{i}, Z_{j}\right)=0$. Otherwise, there is a map $0 \neq g \in \operatorname{Hom}_{B}\left(\tau_{B}^{-} Z_{j}, Z_{i}\right)$. Then we get a cycle

$$
\begin{aligned}
X \rightarrow \tau_{B}^{-} Y_{j} \rightarrow \cdots \rightarrow \tau_{B}^{-} Z_{j} \stackrel{g}{\rightarrow} Z_{i} \stackrel{f_{i+1}}{\rightarrow} Z_{i+1} \rightarrow \cdots \\
\cdots \rightarrow Z_{n} \rightarrow \cdots \rightarrow Y_{1} \stackrel{h_{i}}{\rightarrow} X,
\end{aligned}
$$

and a contradiction. This shows that $\bigoplus_{i=0}^{n} Z_{i}$ is a partial cotilting module, which completes the proof.

1.5. We say that $X$ is a predecessor of $Y$ in $\Gamma_{A}$ (and $Y$ a successor of $X$ ) if there is a path $X=Z_{0} \rightarrow Z_{1} \rightarrow \cdots \rightarrow Z_{s}=Y$ in $\Gamma_{A}$.

Proposition. Let $\eta: 0 \rightarrow \tau_{A} X \rightarrow \bigoplus_{i=1}^{s(X)} Y_{i} \rightarrow X \rightarrow 0$ be an almost split sequence such that $Y_{1} \cong Y_{2}$. Then

(a) If $\operatorname{dim}_{k} \tau_{A} X<\operatorname{dim}_{k} Y_{1}$, then $\tau_{A}^{-n} X$ and $\tau_{A}^{-n} Y_{i}(1 \leq i \leq s(X))$ are well defined for all $n \geq 0$. Moreover, $X$ has no injective successors and does not belong to any oriented cycle in $\Gamma_{A}$.

(b) If $\operatorname{dim}_{k} \tau_{A} X>\operatorname{dim}_{k} Y_{1}, \tau_{A}^{n} X$ and $\tau_{A}^{n} Y_{1}$ are well defined for all $n \geq 0$. Moreover, $\tau_{A} X$ has no projective predecessors and does not belong to any oriented cycle in $\Gamma_{A}$.

Proof. (a): Let $\eta: 0 \rightarrow \tau_{A} X \stackrel{\left(h_{i}\right)}{\longrightarrow} \bigoplus_{i=1}^{s(X)} Y_{i} \stackrel{\left(g_{i}\right)}{\longrightarrow} X \rightarrow 0$ be an almost split sequence with $Y_{1} \cong Y_{2}$ and assume that $h_{1}$ is a monomorphism. Then for all $1 \leq i \leq s(X), \operatorname{dim}_{k} Y_{i}<\operatorname{dim}_{k} X$. In particular, no $Y_{i}$ is injective. Since the almost split sequence starting at $Y_{1}$ has $X$ at least twice as a summand of its middle term, then the above argument implies that $X$ is not injective. Moreover, $\operatorname{dim}_{k} \tau_{A} X<\operatorname{dim}_{k} X<\operatorname{dim}_{k} \tau_{A}^{-} X$. We proceed in this way to show that $\tau_{A}^{-n} X$ and $\tau_{A}^{-n} Y_{i}(1 \leq i \leq s(X))$ are well defined for all $n \geq 0$. 
Assume that $Y$ is a minimal injective successor of $X$. Then we find a sectional path $\tau_{A}^{s} X=Z_{0} \stackrel{f_{1}}{\longrightarrow} Z_{1} \stackrel{f_{2}}{\longrightarrow} \cdots \stackrel{f_{t}}{\longrightarrow} Z_{t}=Y$, for some $s \geq 0$. Since there is an irreducible monomorphism $Z_{1} \rightarrow \tau_{A}^{-} Z_{0}$, then $Z_{1}$ is not injective and there is an irreducible monomorphism $Z_{2} \rightarrow \tau_{A}^{-} Z_{1}$. Continuing this way, we find a monomorphism $Z_{t} \rightarrow \tau_{A}^{-} Z_{t-1}$, which is a contradiction.

Let $\Gamma$ be the right stable component of $\Gamma_{A}$ containing $X$. Then $\Gamma$ contains all the successors of $X$ and it has no periodic modules ( $\operatorname{since} \operatorname{dim}_{k} \tau_{A}^{-n} X$ grows with $n \geq 0$ and hence $X$ is not periodic). If $X$ belongs to an oriented cycle in $\Gamma_{A}$, then the cycle belongs to $\Gamma$ and by [10], $\Gamma$ has trivial valuation, a contradiction.

(b): With the notation above, assume that $h_{1}$ is an epimorphism. In particular, $Y_{1}$ is not projective. Since the almost split sequence ending at $Y_{1}$ has $\tau_{A} X$ at least twice as a summand of its middle term, then $\operatorname{dim}_{k} \tau_{A} X<\operatorname{dim}_{k} \tau_{A} Y_{1}$ and $\tau_{A} X$ is not projective. By (a), $\operatorname{dim}_{k} \tau_{A}^{2} X>\operatorname{dim}_{k} \tau_{A} Y_{1}$. We proceed by induction to show that $\tau_{A}^{n} X$ and $\tau_{A}^{n} Y_{1}$ are well defined for all $n \geq 0$.

If, for some $3 \leq j \leq s(X)$, the module $Y_{j}$ is not projective, then $\operatorname{dim}_{k} \tau_{A}^{2} X>$ $\operatorname{dim}_{k} \tau_{A} Y_{j}$. By induction, $\tau_{A}^{n} Y_{j}$ is well defined. The second part of the statement follows from dual arguments to those used in (a).

1.6. The following results of S. Liu are important in the proof of our theorems.

Proposition [9]. Let $0 \rightarrow \tau_{A} X \rightarrow \bigoplus_{i=1}^{s(X)} Y_{i} \rightarrow X \rightarrow 0$ be an almost split sequence in $\bmod _{A}$. Then

(a) Assume there is a sectional path $\tau_{A} X=Z_{0} \rightarrow Z_{1} \rightarrow \cdots \rightarrow Z_{t}$ in $\Gamma_{A}$ with $Z_{t}$ injective. Assume moreover that $s(X)>4$ or that $s(X)=4$ but no $Y_{i}$ is injective $(i=1, \ldots, s(X))$. Then $\tau_{A} X$ has no projective predecessor in $\Gamma_{A}$ and $\tau_{A} X$ does not belong to a finite cycle in $\bmod _{A}$.

(b) If $\tau_{A} X$ belongs to a finite cycle in $\bmod _{A}$, then $s(X) \leq 4$ and in case $s(X)=4$, there is a $Y_{i}(1 \leq i \leq s(X))$, which is projective.

1.7. The following result generalizes [3] quoted in the introduction.

Theorem [9]. Let $\eta: 0 \rightarrow \tau_{A} X \rightarrow \bigoplus_{i=1}^{s(X)} Y_{i} \rightarrow X \rightarrow 0$ be an almost split sequence such that $\tau_{A} X$ has a projective predecessor and $X$ has an injective successor in $\Gamma_{A}$. Then $s(X) \leq 4$ and in case $s(X)=4$, there is a $Y_{i}(1 \leq i \leq 4)$ which is projective and injective.

\section{Proof of the main Results}

2.1. Proof of the Theorem 1. Let $0 \rightarrow \tau_{A} X \rightarrow \bigoplus_{i=1}^{s(X)} Y_{i} \rightarrow X \rightarrow 0$ be an almost split sequence in $\bmod _{A}$ and assume that $Y_{i}$ is directing $(1 \leq i \leq s(X))$. Assume that $\tilde{\rho}(A) \leq \frac{t-1+\sqrt{t^{2}-4 t}}{2}$ for a natural number $t \geq 4$. We assume that $s=s(X) \geq 5$.

Let $B={ }_{B}^{2}(\eta)$. By the proof of (1.4), we may assume that $B$ is a tilted algebra and $X$ belongs to a directing component $\mathcal{C}$ of $\Gamma_{B}$.

By (1.7), we may assume that $X$ has no injective successors in $\mathcal{C}$. Suppose that $Y_{s}$ is injective in $\bmod _{B}$. Since $\tau_{A} X \rightarrow Y_{s}$ is a sectional map, by (1.6), we get that $\tau_{A} X$ has no projective predecessors in $\mathcal{C}$. Moreover, no $Y_{i}, 1 \leq i \leq s-1$, is 
projective. Indeed, if $Y_{1}$ were projective, then

$$
\operatorname{dim}_{k} Y_{1}+\operatorname{dim}_{k} Y_{s}>\operatorname{dim}_{k} \tau_{A} X+\operatorname{dim}_{k} X=\sum_{i=1}^{s} \operatorname{dim}_{k} Y_{i}
$$

a contradiction. By duality, we may assume that one of the following situations holds:

(1) $0 \rightarrow \tau_{B}^{m+1} X \rightarrow \bigoplus_{i=1}^{s} \tau_{B}^{m} Y_{i} \rightarrow \tau_{B}^{m} X \rightarrow 0$ is a well-defined almost split sequence in $\bmod _{B}$, for all $m \geq 0$;

(2) $Y_{s}$ is projective and injective and $0 \rightarrow \tau_{B}^{m+1} X \rightarrow \bigoplus_{i=1}^{s-1} \tau_{B}^{m} Y_{i} \rightarrow \tau_{B}^{m} X \rightarrow 0$ is a well-defined almost split sequence in $\bmod _{B}$, for all $m \geq 1$.

In case (1) we shall prove that $s \leq t$; in case (2), the same proof yields $s-1 \leq t$ and hence the result.

Assume that $Y_{s}$ is not projective (i.e. case (1)). Consider the Grothendieck group $K_{0}(B)$ and the Coxeter matrix $\phi_{B}$ as a linear transformation $\phi_{B}: K_{0}(B) \rightarrow$ $K_{0}(B)$. Since $B$ is tilted, there are a quiver $\Delta$ and a tilting $k \Delta$-module $T$ such that $B=\operatorname{End}_{k \Delta}(T)$. Therefore, there is an isometry $\sigma: K_{0}(k \Delta) \rightarrow K_{0}(B),[L] \mapsto$ $\left[\operatorname{Hom}_{k \Delta}(T, L)\right]-\left[\operatorname{Ext}_{k \Delta}^{1}(T, L)\right]$, where $[Y]$ denotes the class in $K_{0}(B)$ of a $B$-module $Y$. Moreover, $\phi_{B} \sigma=\sigma \phi_{\Delta}$ (see [18]). In particular, $\rho\left(\phi_{B}\right)=\rho\left(\phi_{\Delta}\right)$.

Observe that $\Delta$ contains the subquiver $\Delta\left(s_{1}, \ldots, s_{m}\right)$ formed by one source 0 , sinks $1, \ldots, m$ and $s_{i}$ arrows from 0 to $i$ such that $\sum_{i=1}^{m} s_{i}=s$. Indeed, $\Delta=\mathcal{S}^{o p}$, where $\mathcal{S}$ is the slice in the component $\mathcal{C}$ formed as the full subquiver of $\mathcal{C}$ whose vertices $Z$ are the starting points of sectional paths to $X$. Since $\mathcal{S}$ contains the arrows $Y_{i} \rightarrow X, i=1, \ldots, s$, we get the claim (of course, $m$ is the number of isoclasses among the $\left.Y_{i}\right)$. Moreover, by [20], we have $\rho\left(\phi_{\Delta}\right) \geq \rho\left(\phi_{\Delta\left(s_{1}, \ldots, s_{m}\right)}\right)$.

On the other hand, $\rho\left(\phi_{\Delta\left(s_{1}, \ldots, s_{m}\right)}\right)=\frac{b-2+\sqrt{b^{2}-4 b}}{2}$, where $b=\sum_{i=1}^{m} s_{i}^{2}$.

Hence $\rho\left(\phi_{\Delta\left(s_{1}, \ldots, s_{m}\right)}\right) \geq \frac{s-2+\sqrt{s^{2}-4 s}}{2}=\rho\left(\phi_{\Delta_{0}}\right)$, where

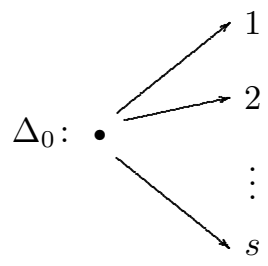

Altogether we get

$$
\frac{t-2+\sqrt{t^{2}-4 t}}{2} \geq \tilde{\rho}(A) \geq \rho\left(\phi_{B}\right)=\rho\left(\phi_{\Delta}\right) \geq \rho\left(\phi_{\Delta_{0}}\right)=\frac{s-2+\sqrt{s^{2}-4 s}}{2} .
$$

Hence $t \geq s$, as desired.

2.2. We recall that the algebra $A$ is said to be tame if for every $d \in \mathbb{N}$ there are finitely many $A-k[t]$-bimodules $M_{1}, \ldots, M_{s}$ which are finitely generated free as right $k[t]$-modules and such that every indecomposable $A$-module $X$ with $\operatorname{dim}_{k} X=$ $d$ is isomorphic to $M_{i} \otimes_{k[t]} S$ for some $1 \leq i \leq s$ and some simple $k[t]$-module $S$.

From now on we will assume that $A$ is a basic connected algebra of the form $A=k Q / I$ (see [6]). The following result follows an idea of von Höhne (see [12]). 
Proposition. Let $A=k Q / I$ be a tame algebra and let $X$ be a non-projective indecomposable module such that the almost split sequence $0 \rightarrow \tau_{A} X \rightarrow \bigoplus_{i=1}^{S} Y_{i} \rightarrow$ $X \rightarrow 0$ has directing middle terms $Y_{i}, i=1, \ldots, s$. Then for all vertices $i \in Q_{0}$ we have

$$
\left|\operatorname{dim}_{k} \tau_{A} X(i)-\operatorname{dim}_{k} X(i)\right| \leq 2 .
$$

Proof. Let $B=B(\eta)$. By (1.3), $B$ is a tame tilted algebra. Then it easily follows that $p \operatorname{dim}_{B} X \leq 1$ and $\operatorname{Hom}_{B}\left(X,{ }_{B} B\right)=0$. Therefore, by [18], $\left[\tau_{A} X\right]=\left[\tau_{B} X\right]=$ $\phi_{B}[X]$.

Consider the Euler (non-symmetric) bilinear form $\langle-,-\rangle: K_{0}(B) \times K_{0}(B) \rightarrow \mathbb{Z}$ defined by $\langle[L],[M]\rangle=\sum_{i=0}^{\infty}(-1)^{i} \operatorname{dim}_{k} \operatorname{Ext}_{B}^{i}(L, M)$ and the corresponding quadratic form $\chi_{B}$. Since $\mathrm{g} \ell \operatorname{dim} B \leq 2$, then $\chi_{B}$ is also the Tits form of $B$ and therefore $\chi_{B}$ is weakly non-negative (see for example [12]). We have $\chi_{B}([X])=1=\chi_{B}\left(\left[P_{i}\right]\right)$, for the indecomposable projective module $P_{i}$ corresponding to the vertex $i \in Q_{0}$. Then

$$
\begin{aligned}
0 \leq \chi_{B}\left([X]+\left[P_{i}\right]\right) & =2+\left\langle\left[P_{i}\right],[X]\right\rangle+\left\langle[X],\left[P_{i}\right]\right\rangle \\
& =2+\operatorname{dim}_{k} X(i)-\left\langle\left[P_{i}\right], \phi_{B}[X]\right\rangle \\
& =2+\operatorname{dim}_{k} X(i)-\operatorname{dim}_{k} \tau_{A} X(i) .
\end{aligned}
$$

Hence, $\operatorname{dim}_{k} \tau_{A} X(i)-\operatorname{dim}_{k} X(i) \leq 2$ and similarly we get the other inequality.

Corollary. Let $A$ be a tame algebra and $\mathcal{C}$ be a directing component of $\Gamma_{A}$. Let $X \in \mathcal{C}$ be such that $\tau_{A}^{m} X$ is well-defined for all $m \geq 0$. Then $\lim _{m \rightarrow \infty} \sqrt[m]{\operatorname{dim}_{k} \tau_{A}^{m} X}=$ 1.

2.3. Proof of the Theorem 2. Let $A$ be a tame algebra and consider an almost split sequence $\eta: 0 \rightarrow \tau_{A} X \rightarrow \overbrace{}^{s(X)} Y_{i} \rightarrow X \rightarrow 0$ such that $Y_{i}$ is directing, $1 \leq i \leq s(X)$.

(a): Assume that $s=s(X) \geq 5$.

Let $B=B(\eta)$ be a tilted algebra by (1.4) and assume that $X$ belongs to a directing component $\mathcal{C}$ of $\Gamma_{B}$. As in (2.1), we may assume that one of the following situations occurs:

(1) $0 \rightarrow \tau_{B}^{m+1} X \rightarrow \bigoplus_{i=1}^{s} \tau_{B}^{m} Y_{i} \rightarrow \tau_{B}^{m} X \rightarrow 0$ is well-defined for all $m \geq 0$;

(2) $Y_{s}$ is projective and injective and $0 \rightarrow \tau_{B}^{m+1} X \rightarrow \bigoplus_{i=1}^{s-1} \tau_{B}^{m} Y_{i} \rightarrow \tau_{B}^{m} X \rightarrow 0$ is well-defined for $m \geq 1$.

Assume that case (1) holds.

Consider $P^{\prime}$ the direct sum of all indecomposable projective modules in $\mathcal{C}$. Let $P^{\prime}=B e^{\prime}$ and $C=B / B e^{\prime} B$ which is a quotient of $A$ by the two-sided ideal generated by an idempotent element. Observe that for $m \geq 1, \tau_{B}^{m} X$ is a $C$-module. Indeed, otherwise an indecomposable direct summand $P$ of $P^{\prime}$ would be a predecessor of $\tau_{B}^{m} X$, for some $m \geq 1$, and hence a predecessor of $\tau_{B} X$, a contradiction to (1.6).

Moreover, $C$ is a tilted algebra. Indeed, consider $\mathcal{S}$ the slice in $\mathcal{C}$ formed by those $Z \in \mathcal{C}$ such that there is a sectional path from $Z$ to $X$ (see [18]). We claim that any indecomposable projective $P$ in $\mathcal{C}$ belongs to $\mathcal{S}$. Indeed, $P$ is a predecessor 
of some $Y_{j}(1 \leq j \leq s)$ because $\bigoplus_{i=1}^{s} Y_{i}$ is a sincere $B$-module. Consider a path $P=Z_{0} \rightarrow Z_{1} \rightarrow \cdots \rightarrow Z_{t}=Y_{j} \rightarrow X$ and assume it is not sectional. Hence for some $1 \leq i \leq t-1, \tau_{B} Z_{i+1}=Z_{i-1}$. We may assume that the modules $Z_{i}, 1 \leq i \leq t$, are not projectives. Then we get a path

$$
P \rightarrow Z_{1} \rightarrow \cdots \rightarrow Z_{i-1} \rightarrow \tau_{B} Z_{i+2} \rightarrow \tau_{B} Z_{i+3} \rightarrow \cdots \rightarrow \tau_{B} Y_{j} \rightarrow \tau_{B} X,
$$

a contradiction. Therefore, $\tau_{B} \mathcal{S}$ is a slice in $\Gamma_{C}$ and $C$ is a tilted algebra.

We shall consider $N=\tau_{B} X$ as $C$-module. Observe that $\tau_{C}^{m} N=\tau_{B}^{m+1} X$ is welldefined. Consider the Grothendieck group $K_{0}(C)$ of $C$ and the Coxeter matrix $\phi_{C}$ as a linear transformation $\phi_{C}: K_{0}(C) \rightarrow K_{0}(C)$. We want to show that for $m \geq 0$, the class $\left[\tau_{C}^{m} N\right] \in K_{0}(C)$ satisfies $\left[\tau_{C}^{m} N\right]=\phi_{C}^{m}[N]$. By [18], it is enough to show that $p \operatorname{dim}_{C} \tau_{C}^{m} N \leq 1$ and $\operatorname{Hom}_{C}\left(\tau_{C}^{m} N,{ }_{C} C\right)=0$ for $m \geq 0$.

Indeed, if $p \operatorname{dim}_{C} \tau_{C}^{m} N>1$, there is a map $0 \neq g \in \operatorname{Hom}_{C}\left(I, \tau_{C}^{m+1} N\right)$ for some indecomposable injective $C$-module $I$. As $B$-module, $I$ is also injective (otherwise, $\tau_{B}^{-} I$ is a predecessor of $\tau_{C}^{m} N=\tau_{B}^{m+1} X$ and $\tau_{B}^{-} I \in \bmod _{C}$ ). Hence there is a map $0 \neq g^{\prime} \in \operatorname{Hom}_{B}\left(Y_{j}, I\right)$ for some $1 \leq j \leq s$, because $\bigoplus_{i=1}^{s} Y_{i}$ is sincere. We get a cycle,

$$
Y_{j} \stackrel{g^{\prime}}{\rightarrow} I \stackrel{g}{\rightarrow} \tau_{C}^{m+1} N=\tau_{B}^{m+2} X \rightarrow \cdots \rightarrow \tau_{B} X \rightarrow Y_{j},
$$

a contradiction. Now, assume $0 \neq g \in \operatorname{Hom}_{C}\left(\tau_{C}^{m} N, \bar{P}\right)$ for some indecomposable projective $C$-module $\bar{P}$. Since $\tau_{B} \mathcal{S}$ is a slice in $\Gamma_{C}$, then $\bar{P}$ is a predecessor of $N$ and as $B$-module, $\bar{P} \in \mathcal{C}$ (use convexity of $\mathcal{C}$, see [15]). Therefore $\bar{P}$ is also projective as $B$-module (otherwise $\tau_{B} \bar{P}$ is defined and $\operatorname{Hom}_{B}\left(P^{\prime}, \tau_{B} \bar{P}\right)=0$, for any projective $P^{\prime}$ in $\mathcal{S}$; hence $\tau_{B} \bar{P} \in \bmod _{C}$, a contradiction). This contradicts (1.6).

Let $\Delta$ be a quiver such that $C=\operatorname{End}_{k \Delta}(T)$ for a tilting module $T$. Consider the isometry $\sigma: K_{0}(k \Delta) \rightarrow K_{0}(C),[L] \mapsto\left[\operatorname{Hom}_{k \Delta}(T, L)\right]-\left[\operatorname{Ext}_{k \Delta}^{1}(T, L)\right]$, which satisfies $\phi_{C} \sigma=\sigma \phi_{\Delta}$. Observe that $T$ does not have preinjective direct summands (since there are no projective modules in the connecting component of $\Gamma_{C}$ ) and since $N$ is a predecessor of a slice in $\Gamma_{C}$, then $N=\Sigma L$ for some preinjective $k \Delta$ module $L$, with $\Sigma=\operatorname{Hom}_{k \Delta}(T,-)$. Hence [18] implies that $\tau_{C}^{m} N=\Sigma \tau_{k \Delta}^{m} L$ and $\left[\tau_{C}^{m} N\right]=\sigma \phi_{\Delta}^{m}[L]$, for $m \geq 0$.

We have the following facts:

(i) $\lim _{m \rightarrow \infty} \sqrt[m]{\operatorname{dim}_{k} \tau_{C}^{m} N}$ exists and equals $\lim _{m \rightarrow \infty} \sqrt[m]{\operatorname{dim}_{k} \tau_{k \Delta}^{m} L}$ (see [12] for a detailed proof).

(ii) $\lim _{m \rightarrow \infty} \sqrt[m]{\operatorname{dim}_{k} \tau_{k \Delta}^{m} L}=\rho\left(\phi_{\Delta}\right)($ see $[5],[15])$.

(iii) $\Delta$ contains a subquiver $\Delta\left(s_{1}, \ldots, s_{m}\right)$ with $s=\sum_{i=1}^{m} s_{i}$ (notation as in 2.1).

By $(2.2), 1=\lim _{m \rightarrow \infty} \sqrt[m]{\operatorname{dim}_{k} \tau_{C}^{m} N}=\rho\left(\phi_{\Delta}\right) \geq \rho\left(\phi_{\Delta\left(s_{1}, \ldots, s_{m}\right)}\right) \geq \frac{s-2+\sqrt{s^{2}-4 s}}{2}$. Therefore $s \leq 4$, contradicting our assumption on $s(X)$.

We get that situation (2) holds, that is, $Y_{s}$ is projective and injective and for all $m \geq 0,0 \rightarrow \tau_{B}^{m+1} X \rightarrow \bigoplus_{i=1}^{s-1} \tau_{B}^{m} Y_{i} \rightarrow \tau_{B}^{m} X \rightarrow 0$ is well defined. Proceeding as above we get that $s-1 \leq 4$. Hence $s(X)=5$ and $Y_{s}$ is projective and injective.

(b): Assume that $s(X) \geq 3$ and that $Y_{1} \cong Y_{2}$. Then by (1.5), we may assume that either case (1) or case (2) in part (a) of the proof holds. In case (1), the argument above yields $1 \geq \rho\left(\phi_{\Delta\left(s_{1}, \ldots, s_{m}\right)}\right)$, with $\sum_{i=1}^{m} s_{i}=s(X)$ and $s_{1} \geq 2$, which 
is impossible since $\rho\left(\phi_{\Delta(2,1)}\right)$ and $\rho\left(\phi_{\Delta(3)}\right)$ are both strictly bigger than 1 . Hence, case (2) holds and $2=s_{1}=s(X)-1$.

2.4. Proof of Theorem 3. Assume $A$ is a cycle-finite algebra, hence tame. Let $X$ be an indecomposable non-projective module and $\eta: 0 \rightarrow \tau_{A} X \rightarrow \bigoplus_{i=1}^{s(X)} Y_{i} \rightarrow X \rightarrow 0$ an almost split sequence. Assume that $s=s(X) \geq 5$. Let $B=B(\eta)$.

If $X$ is not directing, then $X$ belongs to a finite cycle in $\bmod _{A}$. By (1.6), we should have $s \leq 4$. Then $X$ and $\tau_{A} X$ are directing. In case all $Y_{i}(1 \leq i \leq s)$ are directing, Theorem 2 shows that $s=5$.

We may assume that $Y_{1}$ is not directing. By (1.4), there is a sectional path $\tau_{A} X=Z_{0} \rightarrow Z_{1} \rightarrow \cdots \rightarrow Z_{s}$ in $\Gamma_{B}$ with $Z_{s}$ an injective $B$-module. By (1.6), $\tau_{A} X$ has no projective predecessor in $\Gamma_{B}$.

Assume that no $Y_{i}$ is a projective $B$-module, $1 \leq i \leq s$. Then $0 \rightarrow \tau_{B}^{m+1} X \rightarrow$ $\bigoplus_{i=1}^{s} \tau_{B}^{m} Y_{i} \rightarrow \tau_{B}^{m} X \rightarrow 0$ is well-defined for all $m \geq 0$. Consider the sequence $\tau \eta: 0 \rightarrow$ $\tau_{B}^{2} X \rightarrow \bigoplus_{i=1}^{s} \tau_{B} Y_{i} \rightarrow \tau_{B} X \rightarrow 0$. If all $\tau_{B} Y_{i}$ are directing as $B$-modules $(1 \leq i \leq s)$, then by Theorem 2 we are done. Hence by (1.4), we consider the quotient algebra $B_{1}=B(\tau \eta)$ and we get a sectional path $Z_{t}^{\prime} \rightarrow \cdots \rightarrow Z_{1}^{\prime} \rightarrow Z_{0}^{\prime}=\tau_{B} X$ in $\Gamma_{B_{1}}$ with $Z_{t}^{\prime}$ a projective $B_{1}$-module. Since $Z_{t}^{\prime}$ cannot be a projective $B$-module predecessor of $\tau_{A} X$, then $B_{1}$ is a proper quotient of $B$. Consider the sequence $\tau^{2} \eta: 0 \rightarrow \tau_{B}^{3} X \rightarrow$ $\bigoplus_{i=1}^{s} \tau_{B}^{2} Y_{i} \rightarrow \tau_{B}^{2} X \rightarrow 0$. Again either all $\tau_{B}^{2} Y_{i}$ are directing as $B_{1}$-modules and $s \leq 5$ or $B_{2}=B\left(\tau^{2} \eta\right)$ is a proper quotient of $B_{1}$. Repeating the process finitely many steps we end with an algebra $B_{t}=B\left(\tau^{t} \eta\right)$ where all $\tau_{B}^{t} Y_{i}$ are directing. Theorem 2 yields $s=5$ and some $\tau_{B}^{t} Y_{j}$ is projective $(1 \leq j \leq s)$, a contradiction.

We may therefore assume that $Y_{s}$ is a projective $B$-module. By the dual argument, we may suppose that some $Y_{j}(1 \leq j \leq s)$ is an injective $B$-module. As in $(2.1), j=s$. Let $Y_{s}=P_{w}$ be a projective-injective $B$-module. Then the irreducible maps of $\eta$ look as follows:

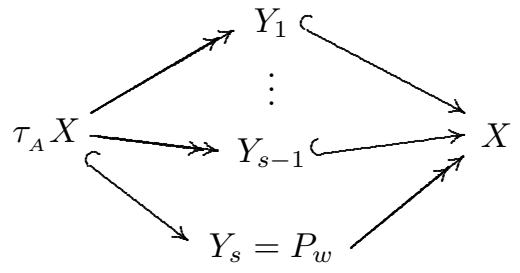

Since $\tau_{A} X$ is directing in $\bmod _{A}$, then $\tau_{A} X(w)=0$. Hence $Y_{i}(w)=0$ for $1 \leq$ $i \leq s-1$. Then $Y_{s}=P_{w}$ is a sincere $B$-module and $B=C[M]=\left(\begin{array}{cc}C & M \\ 0 & k\end{array}\right)$ is a one-point extension of an algebra $C$ by the module $M=\operatorname{rad} P_{w}$. Since $P_{w}$ is injective, also $M=\tau_{A} X$. Hence $\operatorname{rad} P_{w}$ is directing as $A$-module, and therefore $P_{w}$ is directing as $B$-module. We get that $B$ is a tilted algebra and by Theorem 2 we are done. 
2.5 Examples. a) The bound in Theorems 1 and 2 is optimal. Consider $A_{t}$ the algebra given by the quiver $Q$

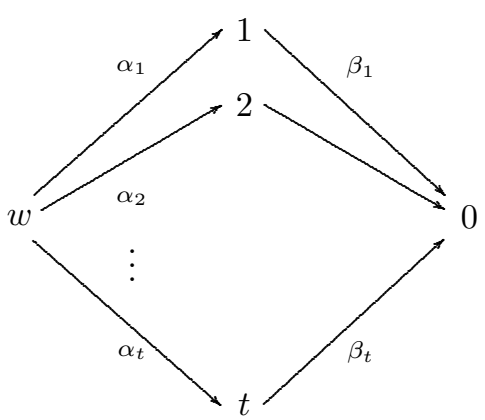

and ideal $I_{t}$ generated by $\sum_{i=1}^{t} \beta_{i} \alpha_{i}$. Then $\tilde{\rho}\left(A_{t}\right)=\frac{t-2+\sqrt{t^{2}-4}}{2}$. There is an almost split sequence as follows:

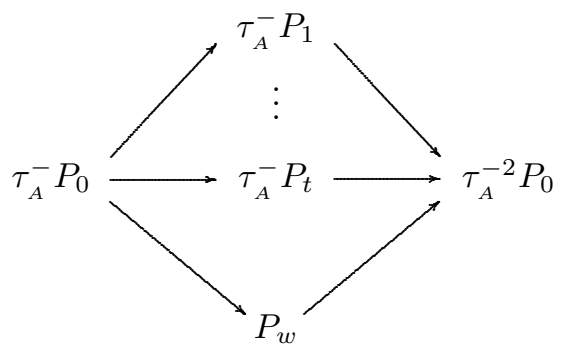

that is, $s\left(\tau_{A}^{-2} P_{0}\right)=t+1$. The algebra $A_{t}$ is tame if and only if $t \leq 3$.

b) Consider $B_{t}$ the algebra given by the above quiver $Q$ and ideal $I_{t}^{\prime}$ generated by $\beta_{i} \alpha_{i}-\beta_{1} \alpha_{1}, i=2, \ldots, t$. Again $\tilde{\rho}\left(B_{t}\right)=\frac{t-2+\sqrt{t^{2}-4}}{2}$. There is an almost split sequence as follows:

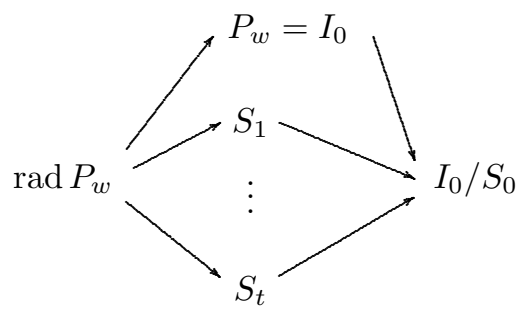

That is, $s\left(I_{0} / S_{0}\right)=t+1$. The algebra $B_{t}$ is tame for $t \leq 4$. 
c) Consider the algebra $C$ given by the quiver

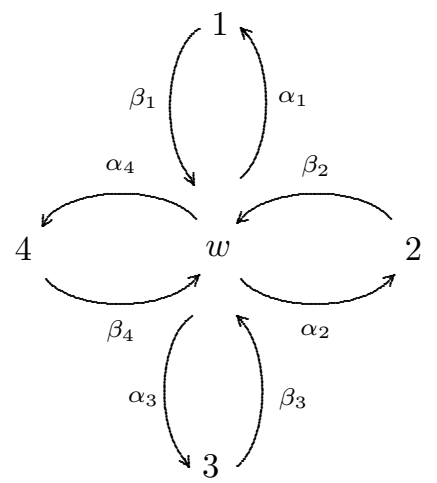

with ideal $I$ generated by $\beta_{i} \alpha_{i}-\beta_{1} \alpha_{1}, i=2,3,4$ and $\alpha_{j} \beta_{i}=0$ for any $1 \leq i, j \leq 4$. Then $C$ has a Galois covering $\tilde{C}$ given by the quiver $\tilde{Q}$

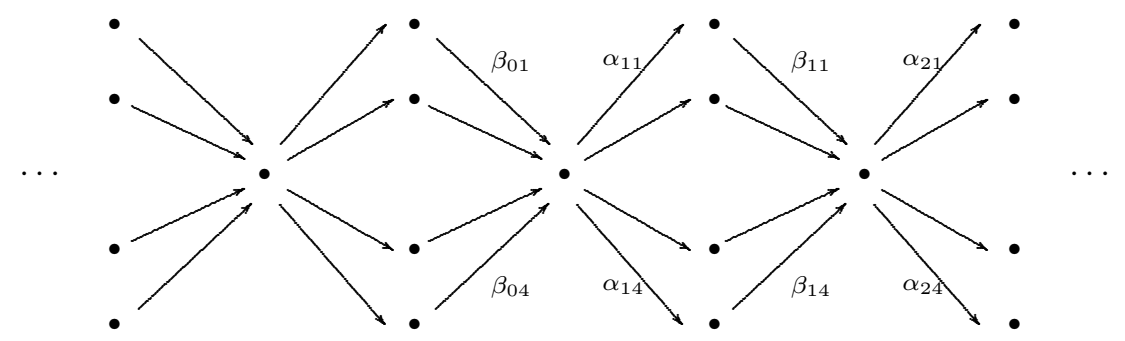

and ideal $\tilde{I}$ generated by $\beta_{j i} \alpha_{j i}-\beta_{j 1} \alpha_{j 1}$ for $i=2,3,4$ and $j \in \mathbb{Z}$ and $\alpha_{j+1, s} \beta_{j, t}=0$ for all $1 \leq s, t \leq 4$ and $j \in \mathbb{Z}$.

It is easy to check that $\tilde{C}$ has no hypercritical or $p g$-critical subcategories and hence $\tilde{C}$ is tame of polynomial growth [19]. Since the covering $\pi: \tilde{C} \rightarrow C$ is defined by the action of the free group $\mathbb{Z}$, then $C$ is tame [13], [19]. There is an almost split sequence in $\bmod _{C}$ of the form

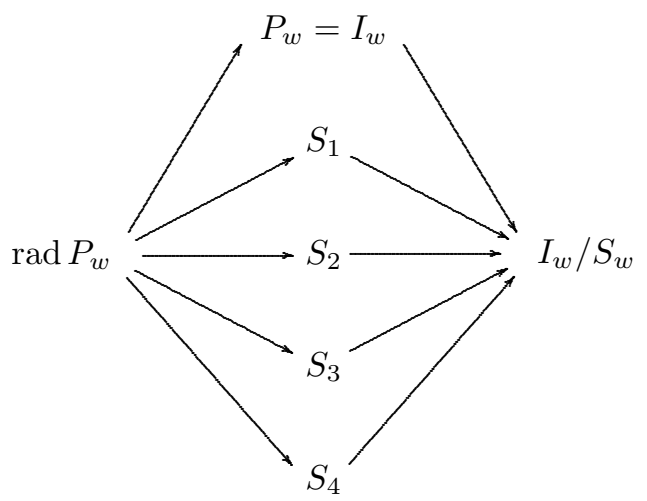

and there are cycles in $\bmod _{C}$ as follows:

$$
S_{i} \rightarrow I_{i} \rightarrow I_{w}=P_{w} \rightarrow P_{i} \rightarrow S_{i}
$$

for all $i=1, \ldots, 4$. 


\section{REFERENCES}

1. I. Assem and A. Skowroński, Indecomposable modules over multicoil algebras, Math. Scand. 71 (1992), 31-61. MR 94c: 16020

2. M. Auslander, I. Reiten and S. Smal $\varnothing$, Representation Theory of Artin Algebras, Cambridge Studies in Advanced Mathematics 36 (1994). MR 98e:16011

3. R. Bautista and S. Brenner., On the number of terms in the middle of an almost split sequence, In Lecture Notes in Mathematics, vol. 903, Springer, (1981), pp. 1-8. MR 83f:16034

4. R. Bautista and S. Smalø, Non-existing cycles, Comm. Algebra 11 (1983), 1755-1767. MR 85d:16010

5. V. Dlab and C. M. Ringel, Eigenvalues of Coxeter transformations and the Gelfand-Kirillov dimension of preprojective algebras, Proc. Amer. Math. Soc. 83 (2) (1981), 228-232. MR 83c: 15007

6. P. Gabriel and A. V. Roiter, Representations of finite-dimensional algebras, Algebra VIII, Encyclopaedia of Math. Sc., vol. 73, Springer, 1992. MR 94h:16001b

7. H. J. von Höhne, On weakly positive unit forms, Comment. Math. Helvetici 63 (1988), 312 336. MR 89g:15033

8. O. Kerner, Tilting wild algebras, J. London Math. Soc. Math. Soc. 39 (1989), 29-47. MR 90d: 16025

9. S. Liu, Almost split sequences for non-regular modules, Fundamenta Mathematicae 143 (1993), 183-190. MR 94g:16018

10. (1993), 405-416. MR 94a:16024

11. J. A. de la Peña, Algebras whose Auslander-Reiten quiver is planar, J. London Math. Soc. (2) 32 (1985), 62-74. MR 87g:16043

12. _ Quadratic forms and the representation type of an algebra, Sonderforschungsbereich Diskrete Strukturen in der Mathematik. Ergänzungsreihe, vol. 003, Bielefeld, 1990.

13. _ Functors preserving tameness, Fundamenta Math. 137 (1991), 177-185. MR 92h:16013

14. J. A. de la Pẽna and M. Takane, Spectral properties of Coxeter transformations and applications, Arch. Math. 55 (1990), 120-134. MR 91k:16015

15. Constructing the directing components of an algebra, Colloquium Math. 74 (1), (1997), 29-46. MR 98d:16026

16. K. Pogorzaly and A. Skowroński, On algebras whose indecomposable modules are multiplicity free, Proc. London Math. Soc. 47 (1983), 463-479. MR 85a:16034

17. C. M. Ringel., The spectral radius of the Coxeter transformation for a generalized Cartan matrix, Math. Ann. 300 (1994), 331-339. MR 95i:17004

18. _ Tame algebras and integral quadratic forms, LNM, vol. 1099, Springer, Berlin, 1984. MR 87f: 16027

19. A. Skowroński, Tame algebras with simply connected Galois covering, Compositio Math. (to appear).

20. M. Takane, The Coxeter transformations of representation infinite quivers, In Proceedings Workshop UNAM 1994, CMS Conference Proceedings 19 (1996), 349-371. MR 97d:16021

Instituto de Matemáticas, UNAM Ciudad Universitaria 04510 México, D. F. México E-mail address: jap@penelope.matem.unam.mx

E-mail address: takane@gauss.matem.unam.mx 